\title{
ANÁLISIS
}

\section{AYUDAS DIRECTAS Y PUBLICIDAD INSTITUCIONAL A MEDIOS DE COMUNICACIÓN EN EL ESCENARIO AUTONÓMICO: INDICADORES Y PAUTAS DE MEJORA}

\author{
Direct subsidies to the media and institutional \\ advertising in Spanish regional laws: Indicators and \\ improvement guidelines
}

\section{Belén Galletero-Campos y Ana-María López-Cepeda}
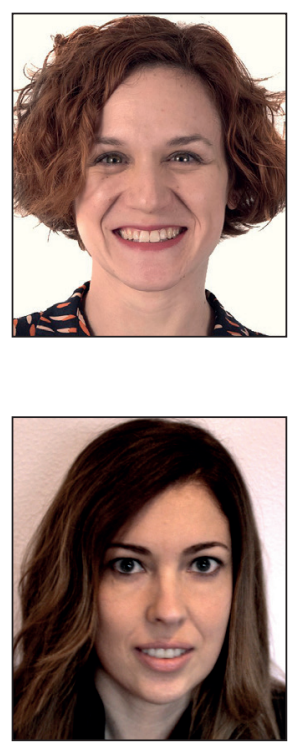

Belén Galletero-Campos es licenciada en Periodismo por la Universidad de Navarra, master en Comunicación Política y Dmpresarial por la Universidad Camilo José Cela y doctora por la Universidad de Castilla-La Mancha (UCLM). Es periodista, ha trabajado en medios de comunicación y es profesora de la Facultad de Periodismo de la UCLM donde forma parte del grupo de investigación MediaCom, dedicado a estudiar la evolución reciente del sistema mediático en la comunidad autónoma de Castilla-La Mancha. Sus intereses de investigación tienen que ver con la calidad, la estructura y el modelo de negocio de los medios locales.

http://orcid.org/0000-0002-9549-9507

belen.galletero@uclm.es

Ana-María López-Cepeda es licenciada en Periodismo y doctora en Comunicación y Periodismo por la Universidad de Santiago de Compostela, así como licenciada en Derecho por la Universidad Nacional de Educación a Distancia. Es profesora en la Facultad de Periodismo de la Universidad de Castilla-La Mancha. Pertenece al grupo de investigación Comunicación Pública: Poder, Derecho y Mensaje y es especialista en políticas de comunicación y estructura de medios, en especial en radiotelevisión pública, transparencia, gobernanza y rendición de cuentas.

http://orcid.org/0000-0001-8328-9142

ana.lopezcepeda@uclm.es

Universidad de Castilla-La Mancha, Facultad de Periodismo Campus Universitario, s/n. 16071 Cuenca, España

\section{Resumen}

La inyección económica de fondos públicos a los medios de comunicación se produce a través de dos vías: subvenciones directas y campañas de publicidad institucional. La finalidad de este artículo es revisar los indicadores que las administraciones autonómicas españolas tienen en cuenta a la hora de conceder ayudas y comprar espacios publicitarios en los medios de comunicación. Cuando estas convocatorias son a menudo reclamadas por el sector, cabe preguntarse si toman en consideración aspectos como la calidad de la información y la responsabilidad social. Para comprobarlo, se analizan las convocatorias de ayudas promovidas por las comunidades autónomas en los últimos diez años (2007-2017), así como la legislación regional vigente en materia de publicidad institucional. A partir de su revisión y de la consulta de una amplia bibliografía científica se diseñan unas pautas para mejorar la justificación de estas vías de financiación.

\section{Palabras clave}

Indicadores; Publicidad institucional; Ayudas públicas; Subvenciones; Medios de comunicación; Responsabilidad social; Legislación; Comunidades autónomas.

\section{Abstract}

The economic contribution of public funds to the mass media takes place in two directions: direct subsidies and institutional advertising campaigns. The purpose of this article is to review the indicators that the Spanish autonomous governments take into account when granting public aid and buying advertising space in the media. When this support is often claimed 
by the profession, it is worth asking if values such as quality of information and social responsibility are considered. To verify this, we analyze the laws about aid for media promoted by the autonomous governments in the last ten years (2007-2017), as well as the current regional legislation on institutional advertising. Based on this review and on the scientific bibliography, we propose guidelines to improve the meaning of these financing channels.

\section{Keywords}

Indicators; Institutional advertising; Subsidies; Media; Social responsibility; Legislation; Spanish autonomous governments; Spanish regions; Laws.

Galletero-Campos, Belén; López-Cepeda, Ana-María (2018). "Ayudas directas y publicidad institucional a medios de comunicación en el escenario autonómico: indicadores y pautas de mejora". El profesional de la información, v. 27, n. 3, pp. 682-691.

https://doi.org/10.3145/epi.2018.may.21

\section{Introducción}

La justificación de las ayudas públicas a medios de comunicación se basa en las orientaciones europeas de "corregir desequilibrios" en base a la "teoría sociopolítica de la responsabilidad social de los medios" (Fernández-Alonso; Blasco-Gil, 2005, p. 182). Las ayudas a la prensa jugaron un papel transcendental durante los años 70 y 80 en Europa debido al "aumento de costes, la caída de ingresos y la reducción de número de títulos" (Humphreys, 2008). No obstante, no se adoptaron de manera homogénea. Se observan tres modelos:

“a) el de los países anglosajones, contrarios a las ayudas porque pueden interferir la libertad de prensa;

b) el de los países nórdicos, que las tienen reguladas como garantías de preservación del pluralismo; y

c) el de los países del Sur europeo, en los que existen de forma directa o indirecta" (Campos-Freire, 2010a, p. 54).

En España, hasta 1983

"las ayudas tenían un marcado carácter discrecional" (Fernández-Alonso; Santana-Cruz, 2000, p. 70-71). [...] La Ley 29/1984, reguladora de la concesión de ayudas a empresas periodísticas y agencias informativas estableció un modelo reglado de ayudas directas e indirectas" (Blasco-Gil, 2008)

con el objetivo de fomentar el pluralismo informativo, considerando a los diarios de menor difusión y a aquellos que contribuyeran a promover la cultura e imagen de España en la esfera internacional. También se regulaban criterios como el consumo de papel y reconversión tecnológica.

Esa normativa estatal fue derogada

"por la Ley 37/1988 como consecuencia de una advertencia de la Comisión Europea" (Campos-Freire, 2010b).

Desde entonces las ayudas públicas a medios de comunicación han pasado a ser competencia de las comunidades autónomas, orientándose en su mayoría hacia medidas de apoyo a las lenguas cooficiales e implementación de nuevas tecnologías. Al mismo tiempo se ha producido un incremento de la publicidad institucional (Campos-Freire, 2010b), entendida como

"aquella comunicación del Estado que se divulga a través de los medios de comunicación (...) a cambio de un desembolso económico por parte de las administraciones públicas" (Cortés-González, 2011).

En España, ésta encuentra su marco jurídico en la Ley 29/2005 de 29 de diciembre de publicidad y comunicación institucional (España, 2005), que nace con la finalidad de

"que toda la información (institucional) llegue a todos los ciudadanos".

Esta norma se complementa con la Ley de contratos públicos (Real decreto legislativo 3/2011) (España, 2011) y con la Ley 19/2013 de 9 de diciembre de transparencia, acceso a la información pública y buen gobierno (España, 2013), considerando la transparencia y el acceso a la información pública como aspectos centrales de toda acción política (Moreno-Sardá; Molina-Rodríguez-Navas; Simelio-Solà, 2017).

Existe una corriente crítica con la financiación de medios a través de ayudas públicas, en especial las dirigidas a la prensa, bajo el argumento de que favorecen la ineficiencia, no abordan los problemas estructurales e incluso pueden dificultar

“la reestructuración necesaria para afrontar el desafío de una creciente competencia entre los distintos medios en la era digital" (Humphreys, 2008).

En España el mercado ha sido el principal elemento regulador sin que el Estado haya podido desarrollar

"un papel interventor y de arbitraje" (Laguna; Martínez, 2013, p. 179),

como sí sucede en otros países europeos.

En los últimos años, fruto de la crisis coyuntural y estructural de los medios (De-Mateo; Bergés; Garnatxe, 2010) y de la caída generalizada de la publicidad ${ }^{1}$, las aportaciones públicas pueden ser relevantes no sólo para acometer mejoras en los medios sino en algunos casos también para garantizar su existencia. Cagé (2016, pp. 81-82) sostiene que numerosas voces

"abogan por una intervención más fuerte del Estado", aunque "aún se interrogan sobre la forma óptima de esa intervención".

Zallo (2011) propone como ejes de las políticas comunicativas la igualdad en el acceso y la comunicación alternativa: la del llamado tercer sector, sin fines comerciales. 
La comunicación social se encuentra a caballo entre la responsabilidad pública y la privada. González-Ballesteros, (2001, pp. 30-31) considera que el Estado tiene que:

"tutelar y proteger el derecho a estar informado que se le reconoce por la Constitución a cualquier ciudadano (...) a fin de evitar que la información continúe siendo un valor perdedor en el mercado salvaje de la comunicación".

Como instrumentos reguladores cita el ordenamiento jurídico, el Consejo de la Comunicación (inexistente en España) o asociaciones de la sociedad civil, entre otros. En este trabajo planteamos que las políticas de comunicación pueden también funcionar como herramientas diferenciadoras en pro de una sociedad mejor informada.

\section{Metodología}

El objetivo de esta investigación es establecer una panorámica de la legislación autonómica en materia de ayudas públicas a medios de comunicación y de publicidad institucional, y comparar la evolución reciente de las cuantías de estas partidas. Sobre la cuestión se han localizado algunas investigaciones, pero los trabajos que abarcan toda o parte de la geografía española están desactualizados (Blasco-Gil, 20082; Feliu-Albaladejo, 2013; Feliu-García; Feliu-Albaladejo, 2016) o se ciñen al estudio de una única autonomía (Fernández-Alonso; Blasco Gil, 2005; Feliu-García; Quintas-Froufe, 2010; Feliu-García; Feliu-Albaladejo, 2011 y 2012; Fernández-Poyatos; Feliu-García, 2012).

A partir de esta revisión, que plantea diferencias significativas entre territorios, nos proponemos conocer los principales indicadores que los gobiernos autonómicos aplican para resolver las asignaciones o la compra de espacios. A día de hoy no existe un compendio reciente de estos indicadores que permita valorar si los cambios estructurales y tecnológicos que han sufrido los medios de comunicación en los últimos años se han visto reflejados en los criterios. Para ello se han localizado las convocatorias y leyes correspondientes a los últimos diez años (2007-2017) en los diarios o boletines oficiales de todas las comunidades autónomas. ción de fondos? El trabajo termina con una propuesta de pautas que contempla valores diferentes a los tradicionales como difusión, tirada, número de usuarios o uso de la lengua propia.

\section{Ayudas públicas}

En la actualidad existen convocatorias de ayudas a los medios de comunicación en nueve comunidades. El sector de la prensa, el más castigado por la crisis, concentra el mayor número. El análisis de las cuantías (gráfico 1 ) muestra que la mayoría se mueve en unos parámetros similares, destacando por encima de la media Galicia y País Vasco. Se ha distinguido entre comunidades con lengua propia y las que no la tienen ${ }^{3}$.

\subsection{Comunidades autónomas con lengua propia}

En estas autonomías se pueden diferenciar dos tipos de ayudas:

- centradas en potenciar mecanismos de identidad cultural y lingüística a través fundamentalmente de medición de audiencias, tiradas, difusión y usuarios únicos;

- basadas además en indicadores empresariales, de innovación y calidad.

Entre las primeras se encuentran las convocatorias promovidas por Galicia, Navarra e Islas Baleares. En Galicia se identifican desde 2007 (Blasco-Gil, 2008) y durante diez años ayudas destinadas a fomentar la presencia del gallego en publicaciones escritas íntegramente en este idioma bajo la competencia de la Secretaría Xeral de Medios. Este organismo también concede desde 1990 y anualmente ayudas económicas basadas en la difusión de la identidad cultural y lingüística del gallego a periódicos impresos (excluidos los gratuitos), empresas radiofónicas privadas y, desde 2000, empresas periodísticas que difundan su actividad informativa en internet.

En Navarra se localizan durante 2007, 2008, 2010 y 2011 convocatorias de ayudas para la utilización del euskera en medios informativos. En 2016 y 2017 se encuentran dos convocatorias de ayudas de la Consejería de Relaciones Ciu-

Se pretende comprobar si la financiación pública revierte en una mejora en la calidad de los medios y del servicio que proporcionan al ciudadano. Las referencias a valores éticos como la defensa de los derechos humanos, la no discriminación, la protección de la juventud y de la infancia, y el respeto al medio ambiente son frecuentes en todas las legislaciones revisadas. Sin embargo, y en lo que concierne a la empresa periodística y al producto informativo, ¿tienen algún impacto aspectos como la sostenibilidad, la calidad y transparencia en la percep-

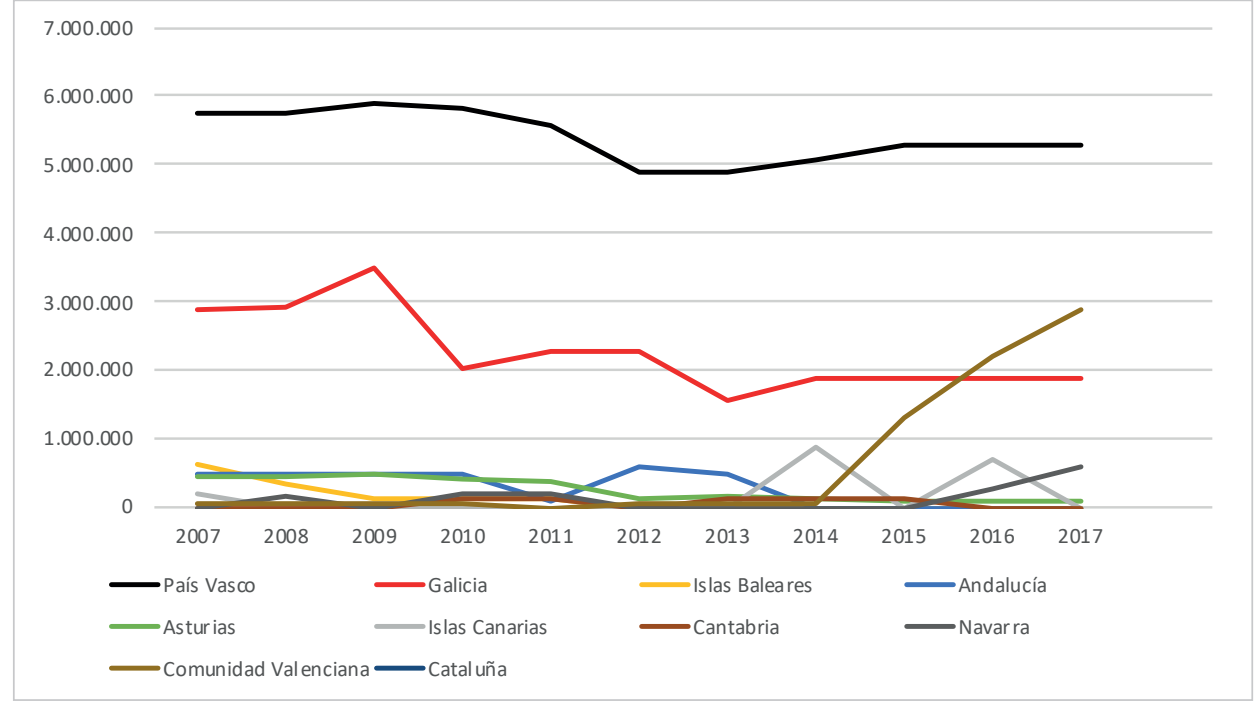

Gráfico 1. Evolución de la cuantía prevista en las convocatorias sobre ayudas públicas a medios de comunicación Fuente: Elaborado a partir de las convocatorias autonómicas de ayudas públicas a medios de comunicación 
dadanas e Institucionales para prensa escrita, radios y medios de comunicación online. Los items más valorados en los años analizados son periodicidad, uso del euskera, impacto $y$, en el caso de los medios online, actualización.

Las ayudas públicas a medios de comunicación otorgadas en Islas Baleares desde 2008 hasta 2011 estuvieron dirigidas a asociaciones sin ánimo de lucro y empresas que editaban publicaciones periódicas no diarias en catalán, emisoras de radio y a la producción audiovisual. Las ayudas se interrumpieron en 2012 y se retomaron en 2016, en esta ocasión destinadas a la prensa impresa o digital diaria en lengua catalana. Se valora la tirada, paginación, distribución, número de secciones o media mensual de visitas a las webs, usuarios únicos y actualizaciones. Para la prensa local y especializada en catalán de periodicidad no diaria un ítem más novedoso es el número de seguidores en las redes sociales.

Existe una corriente crítica con la financiación de medios a través de ayudas públicas bajo el argumento de que favorecen la ineficiencia y no abordan los problemas estructurales

En el segundo grupo de ayudas se localizan aquellas otorgadas en Asturias, donde la Consejería de Cultura y Turismo convoca anualmente subvenciones a medios de comunicación para la normalización social del asturiano y, en su ámbito, del gallego-asturiano. Los indicadores para su reparto se pueden resumir en la elaboración del proyecto presentado, presencia del gallego o asturiano y difusión del medio, criterios ya identificados en 2007 (Blasco-Gil, 2008). Sin embargo, en las cuatro últimas convocatorias se valora también el interés y la calidad del proyecto de acuerdo a aspectos como originalidad, profesionalismo y representatividad social, entre otros.

Las subvenciones que otorga la Comunidad Valenciana provienen de dos organismos: la Acadèmia Valenciana de la Llengua y la Conselleria d'Educació, Investigació, Cultura i Sport. Si bien el uso del valenciano es el principal indicador en ambas normativas, se establecen criterios específicos para cada medio, relacionados con la audiencia y la tirada. En los proyectos digitales se señala la incorporación de contenidos transmedia de producción propia en valenciano. Comunes a todos los soportes son aspectos empresariales puntuables (igualdad, no discriminación o subcontratación con entidades sociales o sin ánimo de lucro).

En Cataluña las ayudas en vigor están dirigidas a los medios de comunicación que publican o emiten $100 \%$ en catalán o aranés. Se valora el cumplimiento del código deontológico de la profesión periodística de Cataluña y los criterios del Consell de la Informació de Catalunya, así como la actualización de contenidos en función de los soportes. A partir de 2016 y 2017 se añade la promoción de un uso no sexista del lenguaje y el respeto a la dignidad de las personas. La apuesta por favorecer el entorno digital se materializa en una cuantía fija de 1.500 euros en concepto de apoyo bási- co por cada medio informativo digital que cumpla todos los requisitos, vigente hasta la última convocatoria (2017). Desde 2007 hasta 2012 se subvencionaron también proyectos dirigidos a consolidar el espacio catalán de comunicación.

El País Vasco es la comunidad donde hay mayor número de ayudas, con una importante evolución a lo largo de los años. De 2007 a 2011 la Consejería de Cultura convocó subvenciones para la realización de estudios de mejora en los medios de comunicación en euskera, destacando criterios como el nivel de financiación propia, la calidad, la difusión (Blasco-Gil, 2008) y la integración de la perspectiva de género. Desde 2014 la Consejería de Cultura y Política Lingüística mantiene una línea de ayudas para incrementar la presencia del euskera en las ediciones digitales de los diarios impresos en castellano y en las agencias de noticias que difunden noticias por internet. Para los primeros se valora el nivel de financiación propia, el volumen de contenido en euskera, la perspectiva de género, el número de clientes y visitas únicas, y la variedad (temática y de soporte). Para las agencias se puntúa el número de noticias y su calidad (diversidad, géneros periodísticos), número de clientes y visitas únicas.

Además desde 2005 la misma Consejería convoca ayudas a publicaciones y medios digitales íntegramente escritos en euskera, y a radios y televisiones cuya difusión sea parcial o total en esta lengua. En estas sí se observa una importante evolución en los indicadores a partir de 2014. Se introduce para todos los soportes estar en posesión del certificado de calidad en la gestión lingüística; para los medios impresos se añade el uso de las nuevas tecnologías para su difusión (smartphones, tablets, aplicaciones...) y la adopción de medidas para la innovación (formación de personal, proyectos de mejora, inmersión en proyectos de investigación). Para los digitales se premia el uso de licencias Creative commons, la originalidad de las noticias y la presencia de mecanismos para favorecer la interacción como Twitter. Paradójicamente se observa que este último indicador ha desaparecido en las convocatorias para 2016, 2017 y 2018, junto con los referentes a la innovación.

Los criterios para otorgar financiación pública suelen aludir a conceptos de carácter interpretativo como la utilidad, la representatividad social o el fomento de los valores democráticos

\subsection{Comunidades autónomas sin lengua propia}

Cantabria, Islas Canarias y Andalucía son las comunidades que, sin disponer de lengua propia, tienen ayudas públicas a medios de comunicación. El Parlamento de Cantabria concedió desde 2010 hasta 2015 ayudas para la creación de espacios y coberturas dedicados a la actividad parlamentaria. En ellas consideraba el ámbito, el nivel de audiencia o tirada y la difusión previa de esta información. Por su composición territorial, en las Islas Canarias el objetivo de las ayudas es compensar los gastos de transporte interinsular en la prensa impresa. 
Andalucía es la región que cuenta con una línea de ayudas más completa. Las convocatorias se suceden desde 2007 y su mayor diferencia es su naturaleza social, poniendo el foco en la mejora del acceso del ciudadano a la información y en el fomento de su capacidad crítica. Los beneficiarios son tanto empresas como entidades sin ánimo de lucro que representen a los profesionales del sector. Aunque al principio se dirigían al fomento de la lectura, desde 2013 se orientan hacia la alfabetización mediática. Se subvencionan los proyectos de difusión de contenido socioeducativo y cultural que sirvan para mejorar el acceso a los medios y comprender con criterio los mensajes, de tal forma que todo ello redunde en una mejora en los niveles de lectura de Andalucía.

Los indicadores han evolucionado desde lo genérico -interés público y calidad del contenido- hasta considerar el nivel de empleo generado, la participación de colectivos vulnerables, el alcance territorial y compromiso medioambiental, el grado de elaboración, la adaptación a las nuevas tecnologías y la promoción de la perspectiva de género, entre otros. Se trata por tanto de apoyar a aquellos medios que trabajan por el empoderamiento de la ciudadanía, recordando que

"ser ciudadano hoy es ser ciudadano en lo mediático" (Gozálvez; Contreras-Pulido, 2014, p. 131).

\section{La cuestión lingüística es la base de bue-} na parte de las ayudas

\section{Publicidad institucional}

El papel controvertido de la publicidad institucional suele vincularse a repartos arbitrarios que favorecen a medios afines, de ahí que los gobiernos hayan trabajado por mejorar sus fórmulas de compra y la transparencia en su gestión. Doce comunidades autónomas han desarrollado legislación autonómica sobre la materia y se suma el proyecto de ley en Galicia, aún sin aprobar, y el Acuerdo marco en Castilla-La Mancha para formalizar contratos con los medios que presenten ofertas. La ley más antigua entre las actualmente en vigor es la de Cataluña, promulgada en diciembre de 2000. A lo largo de más de una década varios parlamentos han aprobado normativas, en orden cronológico: Comunidad Valenciana (2003), Aragón (2003), Navarra (2003), Andalucía (2005), Comunidad de Madrid (2005), Asturias (2006), Canarias (2007), Castilla y León (2009), Baleares (2010) y País Vasco (2010). Las últimas en desarrollar una norma específica han sido Extremadura (2013) y La Rioja (2017).

La información sobre el gasto en campañas no es de fá- cil acceso en todas las autonomías, a pesar de que algunas normas regionales sobre transparencia así lo exigen ${ }^{4}$. Los datos publicados en portales de transparencia muestran que Aragón es la autonomía con mayor gasto en este concepto, y La Rioja y Murcia las que menos invierten. Se produce una reducción importante en el gasto de publicidad institucional en 2012, 2013 y 2014, sin recuperación en la actualidad, en prácticamente todas las comunidades con datos transparentes (gráfico 2).

Aunque la publicidad institucional puede funcionar de facto como mecanismo para apoyar a los medios y mejorar su rentabilidad, como toda acción comercial, está regida por criterios de eficacia comunicativa y precio. Los indicadores que predominan para su distribución son la difusión y la audiencia, así como la oferta económica más ventajosa. La ley andaluza precisa la frecuencia y el coste por impacto, mientras que la catalana declara especial consideración por los medios de comunicación locales y comarcales.

Las leyes más recientes tienen algún indicador relacionado con los nuevos soportes. En La Rioja se incorporan las redes sociales como canal de difusión a todos los efectos y se premia la creatividad y la utilización de nuevas tecnologías. Por otro lado, la Ley de Extremadura presenta como rasgo distintivo la obligación de que todo medio que reciba publicidad institucional esté adherido a la Comisión de Arbitraje, Quejas y Deontología del Periodismo, teniendo en cuenta sus resoluciones a favor o en contra. Además recuerda la necesidad de que los medios desarrollen una "información más visual, didáctica e intuitiva" que pueda ser utilizada por la ciudadanía.

En algunas comunidades se han producido diferencias entre los criterios de adjudicación y su aplicación. En Cataluña en 2006 la distribución de las inserciones de la Generalitat

"entre los distintos diarios no responde exactamente a criterios objetivables de implantación social y difusión" predominando el de la "consideración especial por los medios de comunicación locales y comarcales" (Feliu-García; Feliu-Albaladejo, 2011, p. 62).

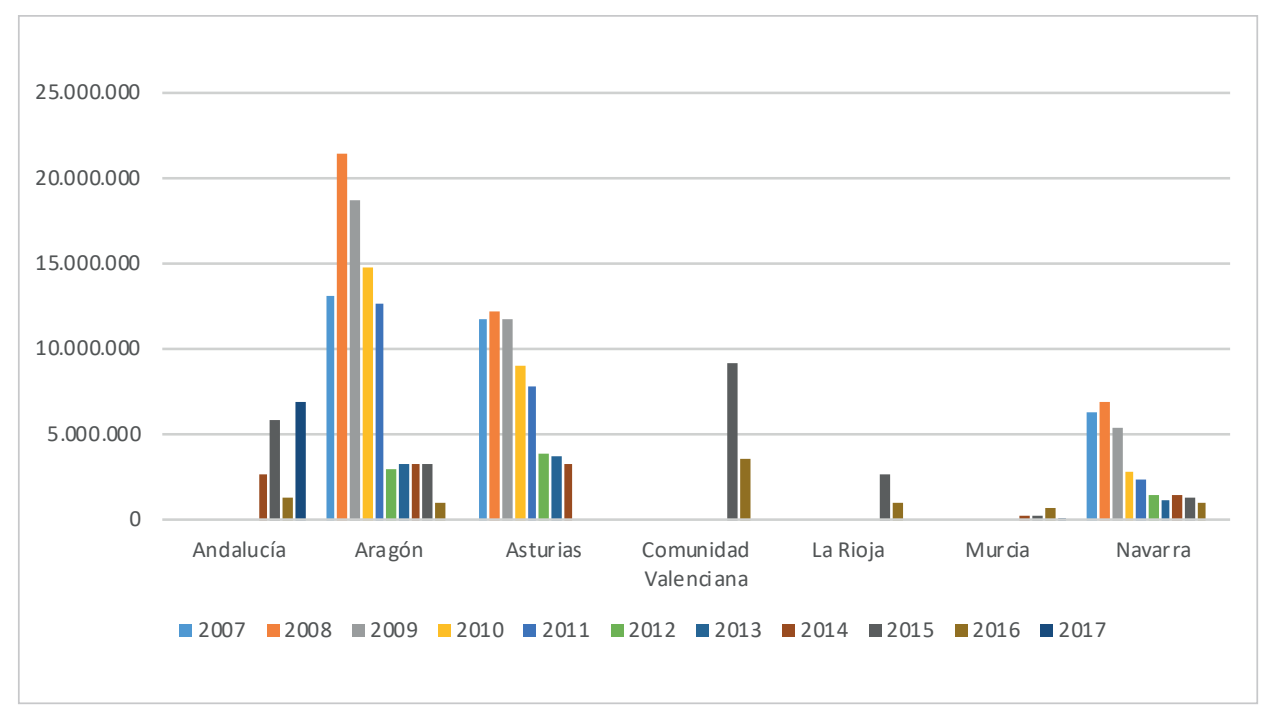

Gráfico 2. Evolución del gasto en publicidad institucional -pública en los portales de transparencia- por comunidades autónomas

Fuente. Elaborado a partir de los datos localizados en los portales de transparencia autonómicos 
En 2009 en Galicia los tres periódicos regionales gallegos de mayor tirada y difusión no figuran entre los principales soportes para las campañas de la Xunta (Feliu-García; Quintas-Froufe, 2010), práctica que estos autores consideran reiterativa en el período 1999-2009. En el mismo año de análisis, en la Comunitat Valenciana:

"la comparación entre la tirada/difusión de los diarios y su grado de utilización como soporte publicitario no se compadece con el objetivo de fijar unos criterios objetivos para la adjudicación de la publicidad institucional que se fijaba el legislador en 2003" (Feliu-Albaladejo; Feliu-García, 2012).

Por otro lado, el Tribunal Superior de Justicia de Castilla y León ha condenado recientemente a la Diputación de León a indemnizar a un medio digital por el reparto de publicidad institucional desde 2011 y hasta 2015 (20minutos.es, 2017).

\section{Apenas se localizan indicadores relativos a} la transparencia y la rendición de cuentas

\section{Pautas de mejora}

El análisis de los items contemplados en las normativas y la revisión bibliográfica nos permite formular una propuesta articulada en tres ejes que contempla 26 indicadores objetivables y no excluyentes a los que ya existen, que podrían incorporarse para conducir a la mejora de la calidad de los medios en los tres aspectos que se plantean. Para la medición de los mismos se propone una metodología mixta que se trabajaría en el marco de las administraciones autonómicas convocantes.

Algunos de los indicadores $(3-7 ; 14-20 ; 22-24)$ se pueden contrastar a partir de una ficha de análisis de contenido de tipo descriptivo validada por la previa codificación de varios investigadores/observadores (Mayntz; Holm; Hübner, 1993). Otros deberían ser certificados por los propios medios mediante declaración jurada a través de la memoria de solicitud. Sería el caso de los indicadores 1, 2, 8-11, 13, 21, 25 y 26.

Todo ello conduce a una puntuación asignada a cada uno de los indicadores o apartados, de acuerdo con el sistema que ya se aplica en la actualidad en las convocatorias y que se ajusta en cada caso según las especificidades de la estructura mediática y del territorio, y los objetivos que se quieran priorizar. Aunque se plantean criterios aplicados a la financiación pública, los elementos sugeridos pueden propor- cionar pautas de mejora en la calidad y en la vocación de servicio de cualquier medio de comunicación, con especial interés en el caso de los digitales.

\subsection{Dimensión ética}

La excelencia y calidad informativa tienen su raíz en la ética profesional (Rodríguez-Martínez et al., 2017). La preocupación por los valores éticos queda patente en las normativas, pero pocas (ley extremeña de publicidad institucional y convocatoria catalana) exigen gestos concretos.

Se propone la adopción del código deontológico de la profesión periodística autonómico o, en su defecto, estatal (Federación de Asociaciones de Periodistas de España, FAPE) y del uso ético de las redes sociales. Algunos aspectos a considerar son (Cruz-Álvarez; Suárez-Villegas, 2017):

- identificación clara de intereses promocionales en las informaciones en formatos como la publicidad nativa;

- respeto al derecho al olvido; y

- solicitud de autorización para el uso de material difundido en redes sociales de un tercero.

Además de la autorregulación profesional, la transparencia informativa y el fomento de la participación del público, articulan la rendición de cuentas (Mauri-Ríos; Ramón-Vegas, 2015). Algunos autores (Redondo-García; Campos-Domínguez, 2016) han advertido de los problemas en la implementación de las reglas sobre transparencia en la propiedad de los medios. Para hacerla efectiva recuperamos el índice empresarial propuesto por De-Pablos-Coello y Mateos-Martín (2004) al que añadimos la estructura de la plantilla, pues es condicionante para valorar la proporción de producción propia.

Como mecanismos internos de rendición de cuentas, caben aquellos más clásicos y los más innovadores como el blog de la redacción o el formulario para advertir errores (Mauri-Ríos; Ramón-Vegas, 2015). Un medio que en la actualidad aplica este último es el Huffington Post a través del botón "Sugiere una corrección". En cualquier caso, en el escenario actual de sobreabundancia de información, la profesión precisa mecanismos que identifiquen las razones

"por las que determinadas informaciones se destacan o se omiten" (García-Avilés, 2016, p. 6).

Se propone considerar las herramientas con vocación de intermediación entre usuarios e instituciones. Un referente es la página Tu derecho a saber, promovida por Civio y en funcionamiento hasta diciembre de 2015. Otra propuesta que

\begin{tabular}{|c|l|}
\hline \multicolumn{2}{|l|}{ Indicadores: Dimensión ética } \\
\hline 1 & Adscripción al código deontológico autonómico o, en su defecto, estatal \\
\hline 2 & Principios éticos y buenas prácticas en el uso de las redes sociales e internet recogidos de forma explícita en un libro de estilo \\
\hline 3 & $\begin{array}{l}\text { Identificación pública en el medio de datos empresariales: tipo de sociedad, composición accionarial, publicación cuenta de resultados, } \\
\text { datos de distribución, estructura redaccional }\end{array}$ \\
\hline 4 & Mecanismos de rendición de cuentas: defensor del lector/blog del editor, botón de corrección, fe de errores (también en el medio digital) \\
\hline 5 & Espacio o herramienta de intermediación entre instituciones y ciudadanía/fomento de la participación \\
\hline 6 & Incorporación de fórmulas de participación (foros de debate, encuentros digitales, contenido aportado por el usuario) \\
\hline 7 & Pluralidad ideológica de los columnistas \\
\hline
\end{tabular}




\begin{tabular}{|c|l|}
\hline \multicolumn{2}{|c|}{ Indicadores: Empresa sostenible } \\
\hline 8 & Empleo generado en el sector en el último año y tipo de contrato \\
\hline 9 & Acreditación de un plan de igualdad \\
\hline 10 & Desarrollo de propuestas alternativas de financiación (micromecenazgo, sistema de membresía, proyectos sin ánimo de lucro) \\
\hline 11 & Alianzas y colaboraciones entre empresas \\
\hline 12 & Contenidos que fomenten la participación de colectivos especialmente vulnerables ${ }^{5}$ \\
\hline
\end{tabular}

se puede adaptar al entorno autonómico es la de MySociety bajo el nombre WhatDoTheyKnow. Ofrece un directorio de autoridades a las que enviar la solicitud y es el gestor (medio de comunicación) quien se encarga de tramitarlas, siendo públicas todas las solicitudes y respuestas. Por otro lado, y respecto al contenido editorial, sugerimos el equilibrio editorial, ya recogido entre los estadísticos planteados por Romero-Rodríguez, De-Casas-Moreno y Torres-Toukoumidis (2017).

\subsection{Empresa sostenible}

La crisis de los medios ha puesto en jaque las fórmulas de negocio tradicionales. Ante la cada vez mayor resistencia al pago por contenido (Vara-Miguel; Negredo; Amoedo, 2017), la concepción de un modelo menos mercantilizado y más social puede ser una alternativa. Algunos indicadores pueden ser:

- fomento del empleo, cuantificado en el número de puestos de trabajo generados en el colectivo y calidad de los mismos;

- incorporación de planes de igualdad;

- promoción de soportes ecológicos.

La diversificación de la financiación y las alianzas o colaboraciones entre empresas (presentes en las convocatorias catalanas de 2008) son vías para incentivar otras fórmulas empresariales que no muestren tanta dependencia de los ingresos publicitarios. Por ejemplo, la edición regional de El diario.es en Castilla-La Mancha ha establecido una colaboración de intercambio no remunerado con otros me- dios locales (Albacete capital, Las noticias de Cuenca) para compartir contenidos exclusivos sin necesidad de contratar corresponsales. Para los medios locales, el acuerdo supone aumentar su visibilidad.

El medio digital cobra cada vez más relevancia, al mismo tiempo que se apuesta por la digitalización y promoción de soportes ecológicos

\subsection{Calidad e innovación}

De acuerdo con Picard (2004), un condicionante de la calidad es la proporción de contenido propio, no sólo el número de items periodísticos actualizados, contemplados en varias convocatorias (Baleares, Navarra). Su evaluación está sujeta a una identificación clara del origen de textos y fotografías mediante la firma. En términos de audiencia y cuando el fenómeno del clickbait (Palau-Sampio, 2016) está disparando las visitas, hay que considerar que el liderazgo puede ser también entendido desde el compromiso del público. Así, se sugiere la valoración de sistemas de membresía, suscripciones al contenido y tiempo medio de lectura. Al respecto, podría invertirse el apartado habitual de noticias más leídas por aquellas que han logrado mayor tiempo de permanencia, en línea con el ranking anual del engagement desarrollado por The New York Times en 2015 (Nafría, 2017). En cuanto a la innovación, se propone la incorporación de

\begin{tabular}{|c|c|}
\hline \multicolumn{2}{|r|}{ Indicadores: Calidad e innovación } \\
\hline 13 & $\begin{array}{l}\text { Audiencia en términos de: } \\
\text { - Visitantes únicos en relación con número total de visitas que reciben todos los solicitantes en el año natural anterior } \\
\text { - Tiempo medio de lectura certificado por entidades externas con datos públicos }\end{array}$ \\
\hline 14 & Ranking de noticias con mayor tiempo de lectura \\
\hline 15 & Suscripciones a newsletter \\
\hline 16 & Perfil activo en redes sociales \\
\hline 17 & Incorporar algún contenido difundido en streaming \\
\hline 18 & Desarrollo de aplicación móvil y suscripciones a la aplicación móvil \\
\hline 19 & Proporción media de contenido diario de elaboración propia respecto al total, acreditado mediante la firma de los artículos/piezas \\
\hline 20 & Incorporación de contenido transmedia \\
\hline 21 & Digitalización de los archivos (prensa, radio y TV) \\
\hline 22 & Sección destinada a periodismo de datos \\
\hline 23 & Implantación de software libre y de código abierto \\
\hline 24 & Uso de Creative commons \\
\hline 25 & Desarrollo de actividades de formación del personal en el último año \\
\hline 26 & Participación activa de, al menos, algún miembro de la plantilla en proyectos de investigación \\
\hline
\end{tabular}


nuevas narrativas y sistemas innovadores de visualización de datos (véase el blog Granada en datos, dentro del medio Granada i Media).

Por último, y a la vista de los cambios experimentados en la producción y la recepción del mensaje periodístico, conviene favorecer la formación de los periodistas o promover su incorporación a proyectos de investigación (convocatoria vasca). Se trata de fomentar la transferencia de conocimiento en la academia y generar una simbiosis necesaria que permita la modernización de los medios. En este sentido, López y Pereira (2007, p. 43) proponen "el fomento de ayudas a los medios que acepten el seguimiento y evaluación de la pluralidad informativa por parte de altos organismos de la comunicación".

El nuevo ecosistema comunicativo permite una mayor implicación de la comunidad, pero es responsabilidad de las administraciones exigir los mecanismos para que se ponga en práctica en los medios

\section{Conclusiones}

En síntesis, las normativas son heterogéneas y su grado de exigencia es variable. La cuestión lingüística es la base de buena parte de las ayudas. Se constata que ha habido cierta evolución en los indicadores a partir de 2012 y que el medio digital cobra cada vez más relevancia, al mismo tiempo que se apuesta por la digitalización y promoción de soportes ecológicos.

Sin embargo, apenas se localizan cuestiones relativas a la transparencia y la rendición de cuentas. Se añade que los criterios aluden con frecuencia a conceptos de carácter in- terpretativo como la utilidad pública, la representatividad social o el fomento de los valores democráticos, cuestiones de difícil cuantificación.

Las pautas aportadas pueden servir de indicativo. Recordemos que los ciudadanos son, en última instancia, quienes financian a través de sus impuestos las políticas públicas de comunicación y, por tanto, su construcción también debería ser pública y participativa (Cortés-González, 2011). El nuevo ecosistema comunicativo permite una mayor implicación de la comunidad, pero es responsabilidad de las administraciones exigir los mecanismos para que se ponga en práctica a través de los medios.

\section{Notas}

1. Según Infoadex, la inversión en medios convencionales ha descendido en 2.750 millones de euros desde 2008 a 2016.

2. Algunas de las convocatorias que estudia Blasco-Gil (2008) en 2007 se mantienen en la actualidad, pero el objetivo de este artículo es conocer la evolución que de ellas y de los indicadores para su otorgamiento se han producido durante los últimos diez años.

3. En Cataluña no se señalan las cuantías, dado que en las convocatorias analizadas se indican que éstas se cuantificarán en base a los presupuestos, sin especificar las cantidades.

4. Se requiere en las normas de transparencia de Andalucía, Aragón, Castilla y León, Cataluña, Extremadura, Murcia, Navarra y País Vasco. Cantabria y Madrid lo exigen en sus proyectos de ley de transparencia

5. La convocatoria andaluza considera como tales aquellos con dificultades de acceso a la prensa como mayores, menores, residentes en zonas de exclusión social, mujeres víctimas de violencia de género, inmigrantes y personas con discapacidad.

\section{Anexo}

\begin{tabular}{|c|c|}
\hline $\begin{array}{l}\text { Comuni- } \\
\text { dad autó- } \\
\text { noma }\end{array}$ & Indicadores en la última convocatoria \\
\hline $\begin{array}{l}\text { Cantabria } \\
(2015)\end{array}$ & $\begin{array}{l}\text { Ámbito de difusión } \\
\text { Nivel de audiencia o tirada } \\
\text { Difusión de la actividad parlamentaria }\end{array}$ \\
\hline $\begin{array}{l}\text { Andalucía } \\
(2016)\end{array}$ & $\begin{array}{l}\text { Interés público } \\
\text { - Nivel de empleo generado } \\
\text { - Fomento de la participación de colectivos vulnerables } \\
\text { - Alcance territorial } \\
\text { - Compromiso medioambiental } \\
\text { Calidad del contenido } \\
\text { - Grado de elaboración } \\
\text { - Adaptación a las nuevas tecnologías } \\
\text { - Contenidos que fomenten la participación social y los valores democráticos } \\
\text { - Contenidos que promuevan la igualdad desde la perspectiva de género }\end{array}$ \\
\hline $\begin{array}{l}\text { Baleares } \\
(2016)\end{array}$ & $\begin{array}{l}\text { Prensa impresa diaria en catalán: ámbito, periodicidad, tirada, paginación y número de islas en las que se distribuye la edición impresa } \\
\text { Prensa digital diaria en catalán: media mensual de visitas, de usuarios únicos, número medio de items periodísticos actualizados por } \\
\text { día y número medio de secciones } \\
\text { Prensa local o especializada no diaria en catalán impresa y/o digital: Tirada, paginación, ámbito, periodicidad, edición en formato } \\
\text { electrónico de la publicación impresa, presencia en plataformas de distribución, suscriptores, media de visitas y usuarios únicos, } \\
\text { actualización y el número de seguidores en redes sociales }\end{array}$ \\
\hline $\begin{array}{l}\text { Canarias } \\
(2016)\end{array}$ & Costes de transportes derivados de la distribución de productos editoriales y prensa \\
\hline
\end{tabular}




\begin{tabular}{|c|c|}
\hline $\begin{array}{l}\text { País Vasco } \\
(2016-2017)\end{array}$ & $\begin{array}{l}\text { Periódicos que publican en castellano: nivel de financiación propia, contenido en euskera, perspectiva de género, número de clien- } \\
\text { tes, visitas únicas y variedad temática y de soporte } \\
\text { Agencias de noticias: número de noticias, crónicas personalizadas, reportajes testimonio, diversidad de las noticias, número de } \\
\text { clientes y visitas únicas } \\
\text { Certificado de calidad en la gestión lingüística } \\
\text { Soporte digital: uso de Creative commons, originalidad de las noticias y mecanismos para favorecer la interacción }\end{array}$ \\
\hline $\begin{array}{l}\text { Asturias } \\
(2017)\end{array}$ & $\begin{array}{l}\text { Grado de elaboración, detalle y concreción del proyecto presentado } \\
\text { Grado de transversalidad en la temática del proyecto } \\
\text { Adecuación a los objetivos generales de normalización del asturiano } \\
\text { Presencia del asturiano o gallego-asturiano en la programación del medio } \\
\text { Interés y calidad del proyecto. } \\
\text { Grado de corrección lingüística en la realización del proyecto }\end{array}$ \\
\hline $\begin{array}{l}\text { Cataluña } \\
\text { (2017) }\end{array}$ & $\begin{array}{l}\text { Uso del catalán o aranés } \\
\text { Cumplir el Código deontológico de la profesión periodística } \\
\text { Actualización } \\
\text { Volumen de producción propia } \\
\text { En el caso de las revistas digitales: } \\
\text { - Deberán ser ediciones nativas para dispositivos móviles (tabletas y smartphones) } \\
\text { - App como mínimo en dos plataformas de difusión } \\
\text { - Fecha fija y periódica de cierre y publicación } \\
\text { - Deberán tener un contenido mínimo equivalente a } 16 \text { páginas de papel DIN A4 }\end{array}$ \\
\hline $\begin{array}{l}\text { Comunidad } \\
\text { Valenciana } \\
(2017)\end{array}$ & $\begin{array}{l}\text { Uso del valenciano en porcentaje } \\
\text { Periodicidad, número de ejemplares, horas de emisión } \\
\text { Prensa impresa: edición de un suplemento semanal de } 10 \text { páginas en valenciano } \\
\text { Prensa digital: contenidos transmedia de producción propia en valenciano } \\
\text { Acreditación de un plan de igualdad } \\
\text { Creación de puestos de trabajo para personas con discapacidad igual o superior al 33\% } \\
\text { Subcontratación con entidades sociales o sin ánimo de lucro } \\
\text { Empresa esté calificada como de inserción sociolaboral }\end{array}$ \\
\hline $\begin{array}{l}\text { Galicia } \\
(2017)\end{array}$ & $\begin{array}{l}\text { Difusión, el empleo regular y la normalización del gallego } \\
\text { Difusión de la identidad y cultura gallegas }\end{array}$ \\
\hline $\begin{array}{l}\text { Navarra } \\
(2017)\end{array}$ & $\begin{array}{l}\text { Periodicidad } \\
\text { Uso del euskera } \\
\text { Impacto } \\
\text { Actualización }\end{array}$ \\
\hline
\end{tabular}

\section{Referencias}

20minutos.es (2017). "La Diputación de León es condenada por discriminar a un medio en el reparto de publicidad". 20minutos.es, 06 de febrero.

https://goo.gl/nEkis7

Blasco-Gil, José-Joaquín (2008). "Las ayudas públicas a la prensa de las Comunidades Autónomas españolas en 2007: tipología, cuantía de las subvenciones y sistemas de adjudicación". Telos, n. 75.

https://goo.gl/28ZRPK

Cagé, Julia (2016). Salvar los medios de comunicación. Barcelona: Anagrama. ISBN: 9788433963963

Campos-Freire, Francisco (2010a). "Gestión de la transición del cambio mediático". En: Campos-Freire, Francisco. El cambio mediático. Sevilla: Comunicación Social, pp. 17-58. ISBN: 9788492860265

Campos-Freire, Francisco (2010b). "Las empresas de medios de comunicación revisan y amplían sus modelos de negocio". Razón y palabra, n. 74.

https://goo.gl/2V8c2x

Cortés-González, Alfonso (2011). "La publicidad institucional en España. Una década en perspectiva”. Razón y palabra, n. 75. https://goo.gl/bpAojK

Cruz-Álvarez, Jesús; Suárez-Villegas, Juan-Carlos (2017). "Pautas deontológicas para el periodismo digital". El profe- sional de la información, v. 26, n. 2, pp. 249-254. https://doi.org/10.3145/epi.2017.mar.11

De-Mateo, Rosario; Bergés, Laura; Garnatxe, Anna (2010). “Crisis, ¿qué crisis? Los medios de comunicación: empresas y periodismo en tiempos de crisis". En: Campos-Freire, Francisco. El cambio mediático. Sevilla: Comunicación Social, pp. 75-106. ISBN: 9788492860265

De-Pablos-Coello, José-Manuel; Mateos-Martín, Concha (2004). "Estrategias informativas para acceder a un periodismo de calidad, en prensa y TV". Ámbitos, n. 11-12, pp. 341-365.

https://www.ull.es/publicaciones/latina/ambitos/11-12/ archivos11_12/coello.pdf

España (2005). “Ley 29/2005, de 29 de diciembre, de publicidad y comunicación institucional". BOE, n. 312, 30 diciembre, pp. 42902-42905.

https://www.boe.es/buscar/doc.php?id=BOE-A-2005-21524

España (2011). "Real decreto legislativo 3/2011, de 14 de noviembre, por el que se aprueba el texto refundido de la Ley de contratos del sector público". BOE, n. 276, 16 noviembre, pp. 117729-117914.

https://www.boe.es/buscar/doc.php?id=BOE-A-2011-17887

España (2013). "Ley 19/2013, de 9 de diciembre, de transparencia, acceso a la información y buen gobierno". BOE, n. 295, 10 diciembre, pp. 97922-97952.

https://www.boe.es/buscar/doc.php?id=BOE-A-2013-12887 
Feliu-Albaladejo, Ángeles (2013). Publicidad institucional y procesos electorales en España (1999-2009). Tesis doctoral, Universidad de Alicante.

http://rua.ua.es/dspace/handle/10045/53555

Feliu-Albaladejo, Ángeles; Feliu-García, Emilio (2012). "Publicidad institucional en la Comunidad Valenciana: la Generalitat como anunciante". En: Peña-Jiménez, Palma; Pacheco-Rueda, Marta; Martínez-Pastor, Esther (eds.). Comunicación institucional y política. Madrid: Fragua. ISBN: 9788470744983

http://rua.ua.es/dspace/handle/10045/24041

Feliu-García, Emilio; Feliu-Albaladejo, Ángeles (2011). "La publicitat institucional a Catalunya". Comunicació: Revista de recerca i d'anàlisi, v. 28, n. 2, pp. 47-67.

http://revistes.iec.cat/index.php/TC/article/view/54802

Feliu-García, Emilio; Feliu-Albaladejo, Ángeles (2016). “Publicidad institucional y cambio de gobierno". En: I Congreso internacional comunicación y pensamiento. comunicracia y desarrollo social, Sevilla. ISBN: 9788494524325 http://rua.ua.es/dspace/handle/10045/59467

Feliu-García, Emilio; Quintas-Froufe, Natalia (2010). “La Xunta de Galicia como anunciante". En: XII Foro universitario de investigación en comunicación. La comunicación institucional. Estado actual. Madrid, 25-26 noviembre. http://rua.ua.es/dspace/handle/10045/25061

Fernández-Alonso, Isabel; Blasco-Gil, José-Joaquín (2005). "Las ayudas públicas a la prensa en Cataluña (2000-2003)". Sphera pública, n. 5.

http://www.portalcomunicacion.com/both/opc/sphera.pdf

Fernández-Alonso, Isabel; Santana-Cruz, Fernanda (2000). Estado y medios de comunicación en la España democrática. Madrid: Alianza. ISBN: 9788420667607

Fernández-Poyatos, María-Dolores; Feliu-García, Emilio (2012). "La publicidad institucional de la Junta de Andalucía". Pensar la publicidad. Revista internacional de investigaciones publicitarias, v. 6, n. 1.

http://revistas.ucm.es/index.php/PEPU/article/view/38659

García-Avilés, José-Alberto (2016). “La información ya no es lo que era. Diez propuestas sobre el periodismo en la sociedad Red". Revista de la Asociación Española de Investigación de la Comunicación, n. 3, pp. 1-8.

http://www.revistaeic.eu/index.php/raeic/article/view/114

González-Ballesteros, Teodoro (2001). "La comunicación social: ¿responsabilidad pública o privada?”. En: Fernández-García, Tomás; García-Rico, Agustín (coords.). Medios de comunicación, sociedad y educación. Cuenca: Ediciones de la Universidad de Castilla-La Mancha, pp. 17-32. ISBN: 8484270769

Gozálvez, Vicent; Contreras-Pulido, Paloma (2014). "Empoderar a la ciudadanía mediática desde la educomunicación". Comunicar, v. 21, n. 42, pp. 129-136.

https://goo.gl/9NyAdb

Humphreys, Peter (2008). "Subvenciones a la prensa en Europa. Una visión histórica". Telos, n. 75.

https://goo.gl/XHn4mH

Laguna, Antonio; Martínez, Francesc-Andreu (2013). “De la dictadura al mercado: la transición de los medios de comu- nicación en España o dónde quedó la responsabilidad social de los medios". Trípodos, n. 32, pp. 171-184.

https://goo.gl/YymDN2

López, Xosé; Pereira, Xosé (2007). La prensa ante el reto en línea. Entre las limitaciones del modelo tradicional y las incógnitas de su estrategia digital. Fundación Alternativas. ISBN: 9788496653801

https://goo.gl/Y1ubW4

Mauri-Ríos, Marcel; Ramon-Vegas, Xavier (2015). “Nuevos sistemas de rendición de cuentas de la información periodística. Exploración del escenario online español". El profesional de la información, v. 24, n. 4, pp. 380-389.

https://doi.org/10.3145/epi.2015.jul.04

Mayntz, Renate; Holm, Kurt; Hübner, Peter (1993). Introducción a los métodos de la sociología empírica. Madrid: Alianza editorial. ISBN: 9788420621319

Moreno-Sardà, Amparo; Molina-Rodríguez-Navas, Pedro; Simelio-Solà, Núria (2017). "Impacto de la legislación sobre transparencia en la información publicada por las administraciones locales". El profesional de la información, v. 26, n. 3, pp. 370-380.

https://doi.org/10.3145/epi.2017.may.03

Nafría, Ismael (2017). La reinvención de The New York Times: Cómo la "dama gris" del periodismo se está adaptando (con éxito) a la era de los móviles. Texas: Centro Knight para el Periodismo en las Américas. ISBN: 9781544822792

Palau-Sampio, Dolors (2016). "Metamorfosis de la prensa de referencia en el contexto digital: clickbait y estrategias de tabloide en Elpais.com". Communication \& society, v. 29, n. 2, pp. 63-80. https://goo.gl/1Qamxh

Picard, Robert G. (2004). "Commercialism and newspaper quality". Newspaper research journal, v. 21, n. 1, pp. 54-66. https://doi.org/10.1177/073953290402500105

Redondo-García, Marta; Campos-Domínguez, Eva (2016). "La transparencia mediática como mecanismo de autorregulación: análisis de su presencia en las webs de los principales medios españoles". Ámbitos, n. 32.

http://institucional.us.es/ambitos/?p=2407

Rodríguez-Martínez, Ruth; López-Meri, Amparo; Merino-Arribas, Adoración; Mauri-Ríos, Marcel (2017). “Instrumentos de rendición de cuentas en España. Análisis comparativo en Cataluña, Galicia, Madrid y Valencia". El profesional de la información, v. 26, n. 2, pp. 255-266. https://doi.org/10.3145/epi.2017.mar.12

Romero-Rodríguez, Luis M.; De-Casas-Moreno, Patricia; Torres-Toukoumidis, Ángel (2016). "Dimensiones e indicadores de la calidad informativa en los medios digitales". Comunicar, n. 49, pp. 91-100.

https://doi.org/10.3916/C49-2016-09

Vara-Miguel, Alfonso; Negredo, Samuel; Amoedo, Avelino (2017). Digital news report España 2017. Navarra: Ediciones Universidad de Navarra.

http://www.amic.media/media/files/file_352_1273.pdf

Zallo, Ramón (2011). Estructuras de la comunicación y de la cultura. Políticas para la era digital. Barcelona: Editorial Gedisa. ISBN: 9788497846653 